\title{
Absorption and Emission of Light in Optoelectronic Nanomaterials: the Role of the Local Optical Environment
}

\author{
Alberto Jiménez-Solano ${ }^{\S}$ Juan F. Galisteo-López, H. Míguez**
}

Instituto de Ciencia de Materiales de Sevilla, Consejo Superior de Investigaciones Científicas (CSIC)-Universidad de Sevilla, C/Américo Vespucio 49, 41092 Sevilla, Spain 
ABSTRACT: Tailoring the interaction of electromagnetic radiation with matter is central to the development of optoelectronic devices. This becomes particularly relevant for a new generation of devices offering the possibility of solution processing with competitive efficiencies as well as new functionalities. These devices, containing novel materials such as inorganic colloidal quantum dots or hybrid organic-inorganic lead halide perovskites, commonly demand thin (tens of nanometers) active layers in order to perform optimally and thus maximizing the way electromagnetic radiation interacts with these layers is essential. In this Perspective we discuss the relevance of tailoring the optical environment of the active layer in an optoelectronic device and illustrate it with two real-world systems comprising photovoltaic cells and light emitting devices.

TOC:
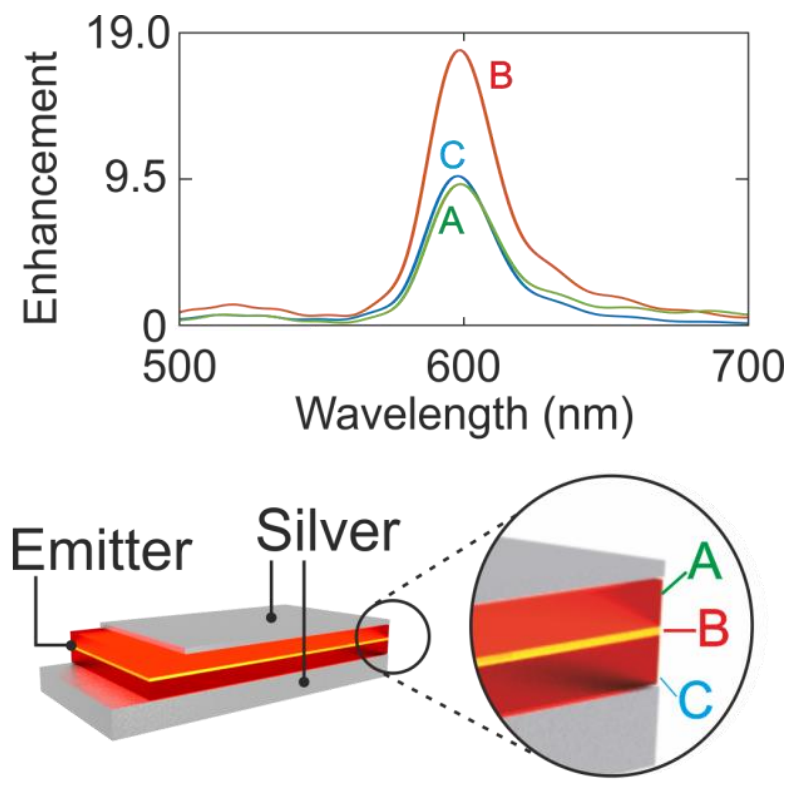
Optoelectronic devices are playing a decisive role in nowadays societal needs in two ways. On the one hand, as a means to produce energy in a sustainable way avoiding the use of fossil fuels, which is achieved by converting solar radiation into electrical current in photovoltaic devices. On the other, they allow minimizing the energetic cost of producing light, where light emitting devices (LEDs) offer the most efficient approach to convert electrical current into electromagnetic radiation. In either of these two complementary routes, converting electromagnetic radiation into electrical current or vice versa, the interaction of light and matter is at the heart of the process and thus maximizing it is central to design highly efficient devices.

When optimizing these systems one must pay attention to the different processes involved in the conversion of light into current. These can be divided into: a proper exit/entrance path of light from/to the device, optimized charge carrier transport and tailoring the optical environment of the active layer. Regarding the flow of light into/out of the device, reducing reflection at its surface or promoting total internal reflection from its interior are traditional approaches in the field of photovoltaics and emission/detection. Beyond traditional approaches such as randomly texturing the surface or introducing anti-reflection coatings, coupling micro and nano-structures to the device has been a widely explored route over the past decade. [1-3] While in some cases the role of the micro/nano structure has only the function of improving the in/out coupling of light into the device, in some other cases it also promotes multiple-scattering of light and an enhanced light matter interaction.

Optimizing charge carrier transport comprises several steps such as transport within the photoactive material, the hole and electron selective layers, extraction at the contacts and a precise design of the interfaces between all conductive layers to minimize losses. In doing so a proper choice of energy levels is decisive. Here materials design is of utmost importance. In particular, for the case of solution processed based devices, which offer the possibility to combine roll-toroll mass production with a room-temperature process, a constant search for new materials which allow for a homogeneous coverage and new functionalities such as flexibility is pursued. In this direction, big efforts are being carried out in the synthesis of new molecules for organic 
solar cells,[4,5] flexible electrodes comprising metal nanowires [6,7] or the use of twodimensional (2D) materials [8-11] to name but a few of the explored directions.

Tailoring the optical environment of the optically active layer is a critical point in the design of any device involving the interaction of light and matter, as it strongly determines its emission and absorption properties. [12] This last point, which is commonly overlooked in the design of optoelectronic devices, is becoming critical in the development of a new generation of materials. These comprise solution processed approaches such as inorganic quantum dots (QD) and hybrid organic-inorganic lead-halide perovskites (HOIP) for which LEDs, [13-16] solar cells [17-19] or photodetectors [20,21] are being developed with efficiencies which are rapidly approaching state of the art performances of commercial devices. An established trend in these approximations is the inclusion of a thin layer of the photo-active materials which, in many cases, is in the tens of nanometers range. For QD-based systems the thickness of the active layer is limited by an increase in device resistance and for HOIP a reduction in the amount of material has environmental implications as the best performing ones contain lead. [22] Thus maximizing light-matter interaction within the final device, by a careful design of its optical environment, is a must in order to make the most out of the active layer. In this sense, the best performing device will certainly require a compromise between the appropriate nature and thickness of all the layers in order to satisfy carrier transport and light-matter interaction demands.

In this Perspective we will focus on the relevance of the optical design of optoelectronic devices in order to optimize their efficiency through a proper engineering of the optical environment of the active layer. In particular we will show how carefully tailoring such environment has profound implications on the way light is absorbed or emitted from these systems. After a general discussion illustrated by the position-dependent photoluminescent properties of a thin layer of emissive material within an optical cavity we will tackle two real-world devices which have been recently proposed within the field of inorganic QDs [18] and hybrid organicinorganic perovskites. [13] These two types of solution-processed materials have become increasingly relevant as active layers for a new generation of optoelectronic devices as 
evidenced by the number of publications dealing with their use in solar cells and LEDs (see Fig.1). The way light is absorbed depending on the position of the active layer within a solar cell or, conversely, the angular and spectral properties of an LED dictated by the spatial distribution of the emitters will be discussed. We demonstrate how introducing small variations in the thickness of the layers of the final device can introduce considerable changes in the absorbed/emitted intensity from the active layer. While further considerations have to be taken into account, such as the conduction properties of the final device or the possibility of producing homogeneous films that prevent shunt paths, a careful design of the optical environment of the active layer in emerging optoelectronic technologies will certainly play a decisive role.

(a)

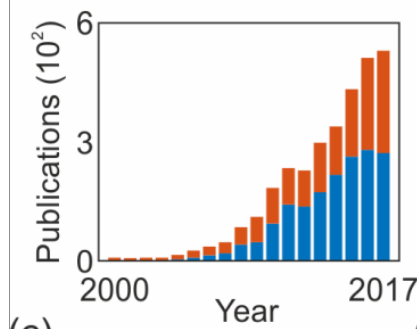

(c)

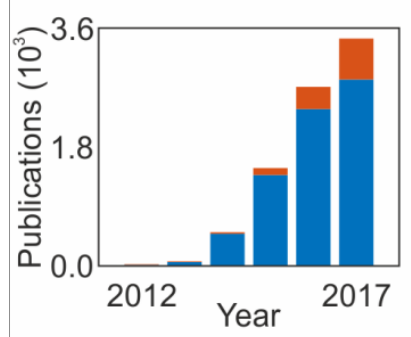

(b)

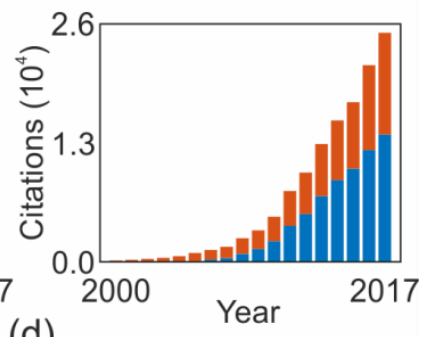

(d)

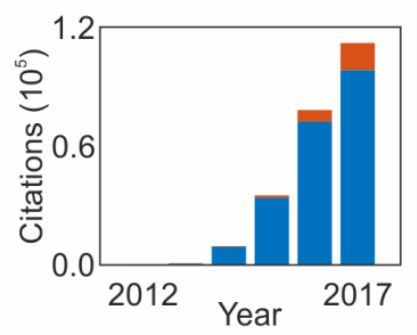

Fig. 1 Number of publications and citations over the past few years dealing with the use of colloidal quantum dots $(a, b)$ and perovskites $(c, d)$ as active layers for the fabrication of solar cells (blue) and LEDs (red bars). Data taken from the Web of Science taking "colloidal quantum dot" and "perovskite" as search terms together with "LED" and "solar cell".

As a means to exemplify the importance of tailoring the optical environment of the active layer in an optoelectronic device let us consider the case of an arbitrarily thin layer of emissive 
material embedded in a $150 \mathrm{~nm}$ thick dielectric cavity with refractive index $\mathrm{n}=1.5$ sandwiched between two metal (silver) films (Fig.2). The PL spectrum of the emissive layer is that of a realistic light source (Lumidot ${ }^{\mathrm{TM}} \mathrm{CdSe} / \mathrm{ZnS} 590 \mathrm{~m}$ QDs from Sigma-Aldrich). Also, one of the metal film covers was considered to be thin enough (thickness $30 \mathrm{~nm}$ ) as to allow the PL to exit the optical resonator. The PL extracted from the whole structure was modelled employing full three-dimensional (3D) simulations performed with a finite-difference in the time-domain (FDTD) commercial code (Lumerical Solutions Inc.). [23] All parameters employed in the FDTD simulations are detailed in the Supplementary Material. The spectral properties were retrieved in two configurations. In one case, from a two-dimensional (2D) detector placed right after the surface of the system over all exit angles; in the other, emission was collected only within a solid angle of $5^{\circ}$ around the normal to the layers. In order to study the effect on the PL of placing the emissive layer at different positions in the resonator, an isotropically emitting dipole (averaging the emission from three identical orthogonally oriented dipoles) was introduced in the modelled structure at different depths with respect to the outer surface.

For the case of a reference free standing emissive layer (Fig.2a) we can already see that emitters placed at different depths of the embedding dielectric layer yield different PL intensities both integrated over all angles (Fig.2b) and within a solid angle of $5^{\circ}$ (Fig.2c). These changes become more pronounced as we introduce the emissive layer within the metal cavity (Fig.2d). Here variations of ca. 50\% in PL intensity can be appreciated depending on the position of the dipole from which we collect the intensity (Fig.2e,f). The observed variations in PL are dictated by two factors. On the one hand the optical environment of the emitter, given by the positiondependent photon local density of states (LDOS), affects the radiative component of the decay rate and thus can improve/quench its quantum yield (QY). [12] In fact, the emissive properties of the active layer within the device are dictated by Fermi's golden rule which for a dipole transition between an initial (i) and a final (f) states gives a fluorescence decay rate:

$$
\Gamma(\boldsymbol{r})=\frac{2 \pi}{\hbar^{2}} \sum_{|f\rangle}|\langle f|\widehat{\boldsymbol{H}}| i\rangle|^{2} \delta\left(E_{f}-E_{i}\right)
$$


(a)

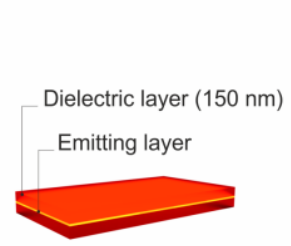

(b)

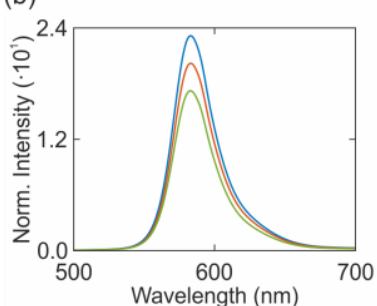

(c)

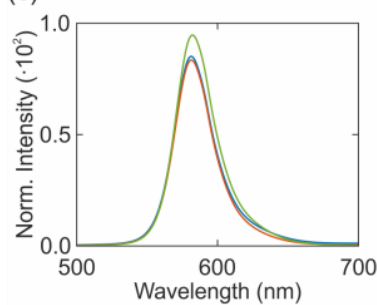

(d)

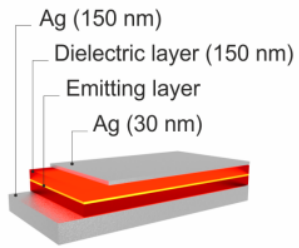

(e)

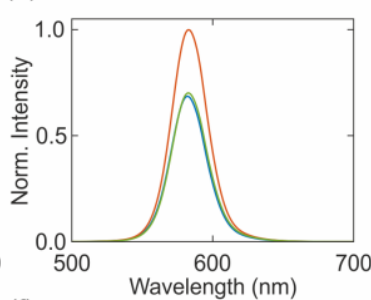

(f)

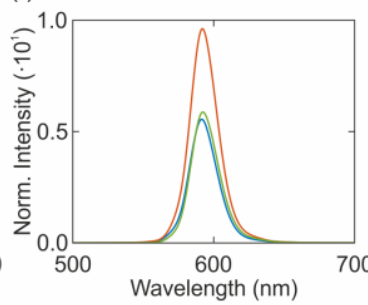

(g)

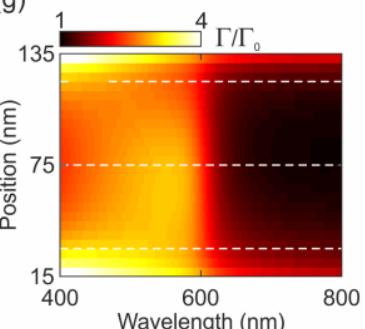

(h)

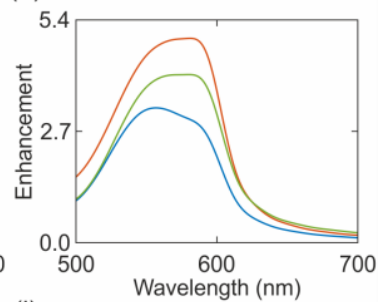

(i)

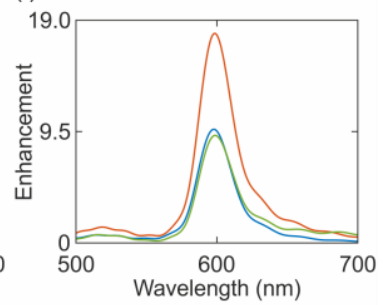

Fig. 2 Numerical simulation of the PL properties. Schematics, (a) and (d), and PL spectra integrated over all angles, (b) and (e), and within a $5^{\circ}$ solid angle, (c) and (f), for both the freestanding film (first column) and the film contained within the metal optical cavity (central column). In both cases, the layer of isotropically emitting dipoles is placed at different depths within the embedding dielectric slab: $120 \mathrm{~nm}$ (green), $75 \mathrm{~nm}$ (red) and $30 \mathrm{~nm}$ (blue curve). (g) Spectral and spatial map of the quotient between the photon LDOS in the dielectric slab $(n=1.5)$ within the resonator and that of the same slab freestanding. $(h)$ and (i) show the ratio of the PL spectra attained from the two configurations under analysis for all exit angles, (h), and for a $5^{\circ}$ cone, (i).

Which, using the interaction Hamiltonian $\widehat{\boldsymbol{H}}=-\widehat{\boldsymbol{\mu}} \cdot \widehat{\boldsymbol{E}}$ can be further expressed as:

$$
\Gamma(\mathbf{r})=\frac{2 \omega_{i f}}{3 \hbar \varepsilon_{0}}|\boldsymbol{\mu}|^{2} \rho\left(\boldsymbol{r}, \omega_{i f}\right)
$$

Where $\mu$ is the dipole moment and $\rho\left(\mathbf{r}_{\mathbf{0}}, \omega_{\mathrm{if}}\right)$ is the LDOS. While these particular expressions deal with a single dipole emitter, Fermi's Golden Rule can also be applied to band to band 
transitions of free carriers in direct gap semiconductors. [24] Please notice that even the homogeneous film shown in Fig.2a presents an uneven photon LDOS, which explains the weak PL intensity variations observed when the emitting thin layer is located at different depths (Figs. $2 \mathrm{~b}$ and $2 \mathrm{c}$ ). This effect is even more intense when the same film is located within a metallic resonator, as in Figs. 2d, 2e and 2f. Any further structuration of such medium will bring about an even stronger spatial and spectral redistribution of the LDOS. A second relevant factor is the out-coupling efficiency of the structure, as, in a film, total internal reflection determines the amount of the radiated power by the dipole that is able to exit the structure, this being smaller as the refractive index contrast between the inner (emitting) and outer (propagating) media increases.

Fig.2g shows a spectral map of the photon LDOS change within the dielectric slab containing the emissive film sandwiched between the two silver mirrors. Results are normalized to the LDOS of a free-standing homogeneous medium of similar refractive index. Here we can see a modulation which qualitatively reproduces the changes that we observe if we take the PL ratios of the three dipole positions with and without the metal layers (Fig.2h and Fig.2i). While the map in Fig.2g points to expected QY changes, only the full simulation provides us with information on the amount of generated radiation which is able to exit the film. Fig. $2 \mathrm{~h}$ and Fig.2i show the actual directional enhancement expected both for the PL integrated over all angles and for that emitted within a $5^{\circ}$ solid angle reaching an external detector. These two graphs evidence that the position of the emitter within the structure will drastically determine not only the enhancement in PL but also the spectral components being enhanced at a given angle. Further, the full simulation allows us to detect the role of the metal layers, which introduce ohmic losses at regions close to the metal boundaries where a large LDOS would lead us to expect enhanced emission (see Fig.S1 in the Supplementary Material).

While the above example offers an intuitive way to decide where to place an optically emissive layer of nanometric dimensions to optimize its emission from a dielectric slab, real world devices usually have a more complex structure with several layers of different thickness and 
optical constants. Here a simulation of the optical environment of the emitter/absorber is mandatory in order to maximize the device efficiency. In the remaining of this Perspective we will consider two real world devices which have been recently reported, dealing with inorganic QDs [18] and hybrid organic-inorganic perovskites, [14] and study how tailoring the optical environment of the active layer can dramatically affect the performance of the device. Here we will only concentrate on the proposed device architecture and introduce slight variations in the thickness of the different layers of the reported structure. Employing a numerical approach combining the Transfer Matrix Method (TMM) and Genetic Algorithms we obtain the most and least favorable structures (changing the thickness of all the layers but the optically active one) and analyze them from the point of view of the way the optically active layer absorbs/emits light. The thickness of all films comprising the studied devices are listed in Tables 1 and 2 of the Supplementary Material.

Regarding its application in photovoltaics, solution processed devices comprising hybrid organic inorganic perovskites are certainly attracting increasing attention due to its meteoric rise in efficiency. [25] Nevertheless in the architectures presenting the highest device efficiency the active perovskite layer is in the hundreds of nanometers range and thus absorption of most the incident solar radiation is already achieved. While for these devices the optical environment of the active layer certainly plays a role, as already shown for $\mathrm{MAPbI}_{3}$ solar cells, [26,27] it is not expected to be as critical as those devices for which much thinner active layers are employed. This is the case for QD-based solar cell devices where, as mentioned above, the active layer cannot be made arbitrarily large. It is in this kind of solution processed devices where one expects the largest impact of the optical environment. Here we consider the structure proposed by Bernechea and co-workers [18] where solar cells based on $\mathrm{AgBiS}_{2}$ colloidal QDs were fabricated yielding a power conversion efficiency of $6.3 \%$. This approach relies on the possibility of employing an active material made from non-toxic abundant elements, which would overcome one of the main limitations of most QD-based solar cells which rely on materials containing heavy metals. [28] Nevertheless the above results can be extended to 
devices relying on more conventional QDs. As mentioned above, while a thick QD film increases the possibility of absorbing the incident solar radiation, it also contributes to increase the resistance of the final device. Hence it becomes relevant to maximize the absorption of the thin (35 $\mathrm{nm}$ in the present case) active layer considered.

The absorbed power density within a given volume of a material is dictated by the normalized electric field intensity at that volume and the optical constants of the material: [29]

$$
P_{a b s}=\frac{1}{2} \omega \varepsilon_{0}(2 n k) \int_{V}|\boldsymbol{E}(\boldsymbol{r})|^{2} d \boldsymbol{r}
$$

where $\omega$ is the angular frequency, $\varepsilon_{0}$ the free-space permittivity, $\mathbf{E}(\mathbf{r})$ is the normalized electric field, $\mathrm{n}$ and $\mathrm{k}$ are the real and imaginary components of the refractive index and $\mathrm{V}$ is the volume occupied by the absorber. In order to determine the role of the optical environment and optimize the structure (from the point of view of light absorption) we have calculated the absorption of the $35 \mathrm{~nm}$ film by using the normalized electric field intensity obtained with a home-made TMM code. This was combined with a Genetic Algorithm in order to, within a 50\% variation in the thickness of all the layers but the active one, obtain the most and least favorable cases. The optical constants of the different materials used in the simulations were taken from refs. 30 (Ag), $31\left(\mathrm{ZnO}\right.$ and $\left.\mathrm{MoO}_{3}\right)$ and 32 (ITO). For $\mathrm{AgBiS}_{2}$ and PTB7 the optical constants were extracted from spectroscopic measurements provided by the authors of Ref.18. Fig.3a shows the structure we used for the numerical simulations (where layer thicknesses were extracted from Ref. 18) and Fig.3b the absorptance weighted over the AM 1.5 solar spectrum in the proposed structure as well as the best/worst case scenarios (detailed thickness of the layers for all three cases can be found in Table 1 of the Supplementary Information). Here it can be appreciated how an overall enhancement in absorption of $4 \%$ is attained in the best case as compared to the proposed structure and $13 \%$ as compared with the worst case by changing the position of the active layer within the final device. 
(a)

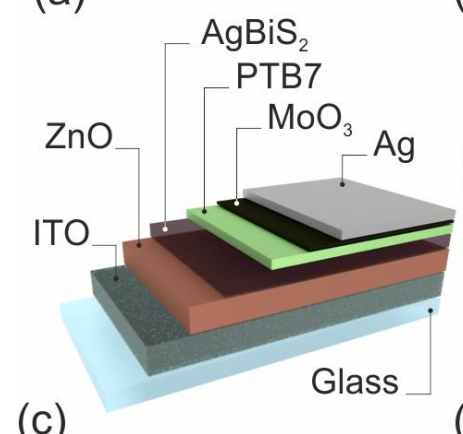

(c)

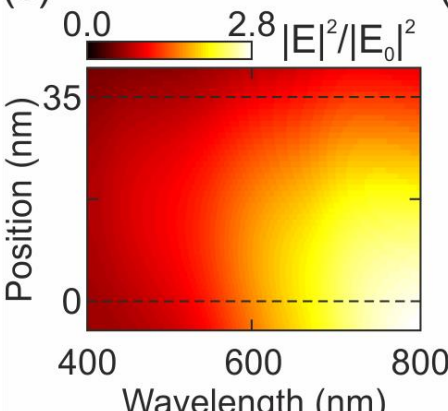

(e)

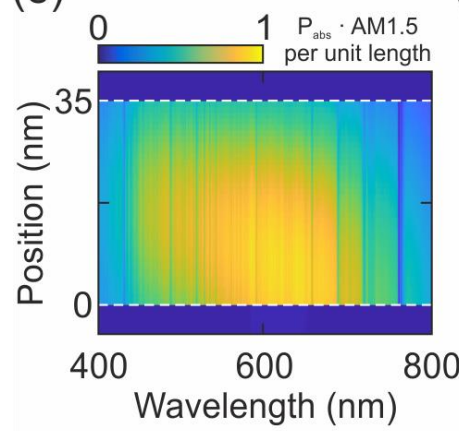

(b)

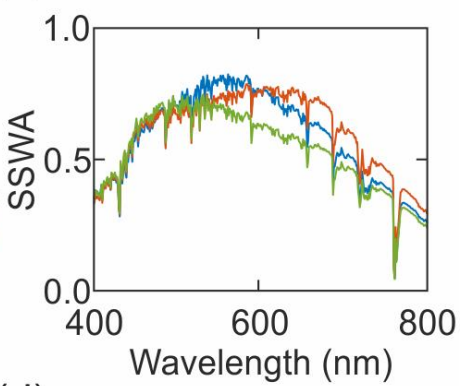

(d)

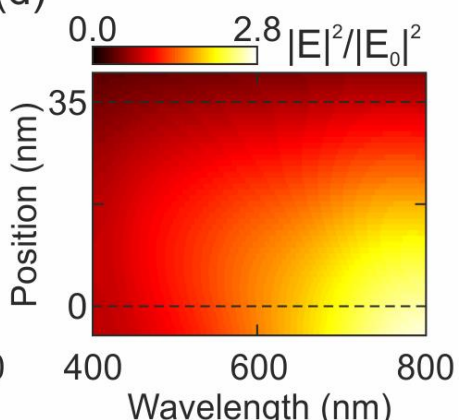

(f)

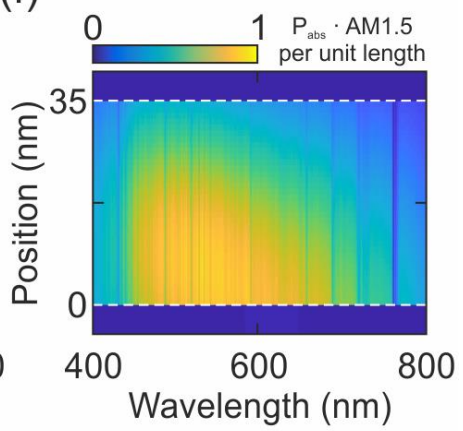

Fig. 3 Numerical simulation of the absorption at the active layer of a $Q D$ solar cell. (a) Shows the simulated device according to Ref. [18]. (b) Solar spectrum weighted absorptance over the whole active layer for the proposed device (blue) and the best/worst scenario cases (red/green curves respectively). (c) and (d) show the total field intensity throughout the $Q D$ layer for the best/worst performing devices respectively. $(e)$ and $(f)$ show the solar spectrum weighted power absorbed per unit length of the same devices. Dashed lines represent the boundaries of the QD layer within the solar cell.

In order to understand these changes we have plotted the spatial and spectral distributions of the normalized light field intensity (Fig.3c,d) and the solar spectrum weighted power absorbed per unit length (Fig.3e,f) for the best and worst case scenarios. Here it becomes evident that altering 
the optical environment (i.e., modifying the thickness of the surrounding layers, in this case) it is possible to maximize the light field intensity within the QD slab, thus increasing the power absorbed by it. This can be readily seen by comparing the absorption profiles along the $35 \mathrm{~nm}$ width of the QD layer attained for the best (Fig.2e) and worst (Fig.2f) case. While further considerations should be taken into account for the optimized multilayer cell structure, such as the resistance of the final device, these results clearly show the relevance of paying attention to the optical environment of the QD layer.

Finally we would like to highlight again the fact that the above design becomes more critical as the thickness of the active layer lies in the tens of nanometer range. For conventional state of the art perovskite solar cells [33] presenting efficiencies above $20 \%$ a similar variation in structural parameters of the device to modify the optical environment of the active perovskite layer yields much smaller variation in its absorptance as shown in Section S4 of the Supplementary Information.

Next we shall consider the role of the optical environment on the active layer of an LED based on hybrid organic-inorganic lead-halide perovskites. [14] Many reports have highlighted the relevance of including a thin film of perovskite, dense or nanocrystalline, as active material in order to overcome their small exciton binding energy and promote radiative recombination through the spatial confinement of the exciton. [34] As a matter of fact, most of the devices presenting the highest efficiencies present active layers with a thickness in the tens of nanometers range (see Section S5 of the Supplementary Information and references therein). This factor adds to the reduced amount of lead present in the device, already mentioned above. As an efficient radiative recombination is crucial for light emission applications, beyond the structural contribution provided by the confinement, a proper engineering of the optical environment also plays a key role as the radiative component of the recombination rate is dictated by the local density of radiative states at the emitter's position, as expressed in equation (1). [12] In this sense, the high efficiency attained in ref. [14], accompanied by an enhanced material stability due to the addition of long-chain ammonium halides in the perovskite 
synthesis can be in principle further improved. For instance, the electroluminescent QY of the active material in the best performing devices was $6.6 \%$ for iodide-containing perovskites and $40.1 \%$ for bromide-containing ones. We have chosen the former as test-bench to study the effect of the optical environment as there is further room for improvement. The optical constants for the simulations performed for this system were taken from Refs. 35 (Al), 36 (LiF), 37 (TPBi), $33\left(\mathrm{CH}_{3} \mathrm{NH}_{3} \mathrm{PbI}_{3}\right), 38$ (PolyTPD) and 32 (ITO).

In order to simulate the effect of the optical environment on the above mentioned device (Fig.4a shows a schematic diagram of the structure) we obtain a first estimate by calculating with our TMM code the product of the total field intensity within the active layer (averaged over all angles of incidence of an external white light plane wave) and the spectral shape of the emission. According to the reciprocity theorem $[39,40]$ this should be a good estimate of how an isotropic source emitting from within the device would couple to propagating modes in the free space. Again a Genetic Algorithm approach is used to vary the thickness of the different layers within $20 \%$ of the original values and extract the best/worst case scenarios (a complete list of layer thicknesses for all three cases can be found in Table 2 of the Supporting Information). Once the two extremes are found, a full 3D FDTD calculation is performed in order to simulate the emissive properties of the active layer. Fig. $4 \mathrm{~b}$ and $4 \mathrm{c}$ show the PL spectra collected over all exit angles and within a $5^{\circ}$ solid angle respectively for the original device and the best and worst performing devices (from the point of view of the optical environment). Here it becomes evident that a rational design of the structure strongly influences the way light is emitted from it. In order to understand such different behaviors we will next discuss the spectral and intensity characteristics of the emission from the active layer depending on the spatial position from which light is emitted. 
(a)

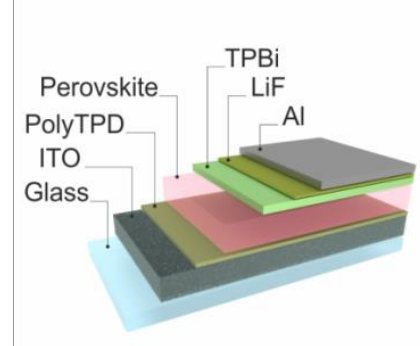

(d)

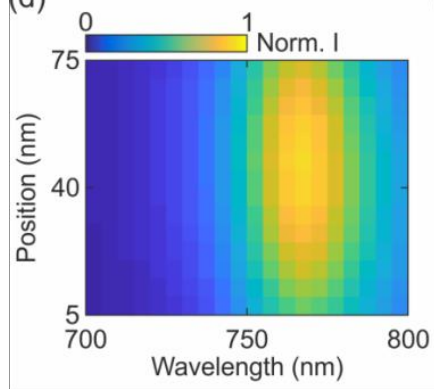

(b)

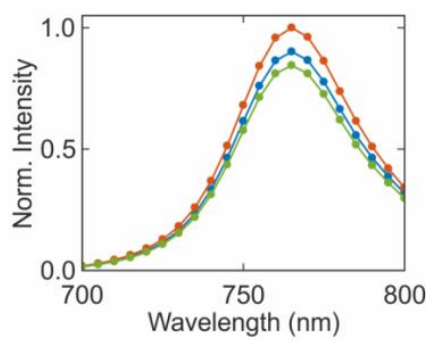

(e)

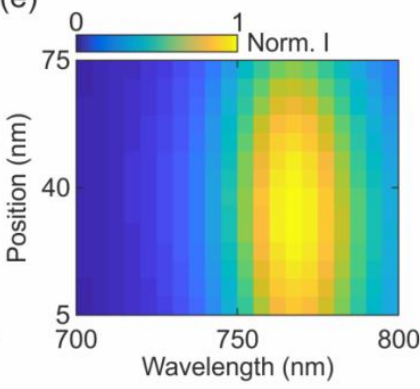

(c)

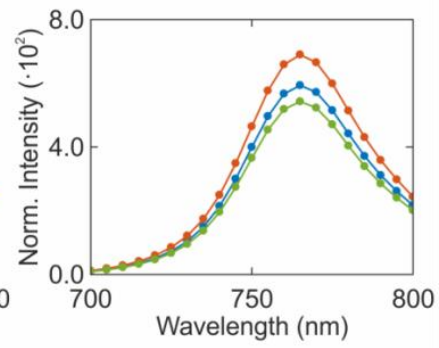

(f)

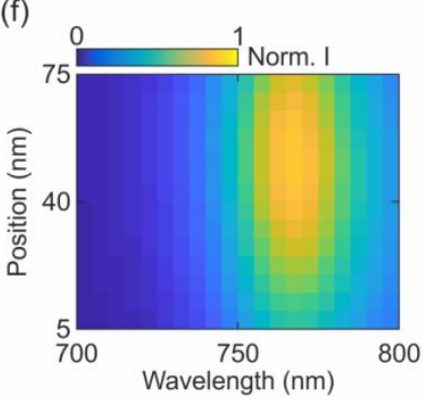

Fig. 4 Numerical simulation of the emission from the active layer of a lead-halide perovskite LED. (a) Shows the simulated device according to Ref. 14. PL spectra from the active layer of the device collected for all exit angles (b) and in a $5^{\circ}$ solid angle (c) for the proposed device (blue) the best case (red) and the worst case (green curve) scenarios. (d-f) PL spectra collected over all exit angles from the LED depending on emitting dipole position for the proposed device, $(d)$, the best, (e), and the worst, (f), performing ones.

Figs. 4d-f show contour plots of the PL spectra collected by an external detector (placed within the glass substrate) from an array of isotropically emitting dipoles located across the active layer, normalized to the case of the best performing configuration. In the simulation all the dipoles are considered identical, assuming that all perovskite nanocrystals are electrically excited with identical efficiency. Also in this case, as it happened for the solar cell studied above, it becomes evident that depending on the thickness of the different layers, the optical environment is modified and hence the emission from the active layer varies. In the device structure proposed in ref. [14] (Fig.4d), the optical environment of the emitters provides the highest emission from those nanocrystals located at the top part of the active layer, closer to the TPBi film. Optimizing the thickness of the different layers comprising the structure (Fig.4e) alters the spectral and spatial distribution of the emission. Here an enhanced emission is coming 
from the appearance of a broad region with higher density of photon modes in the active layer closer to the ITO film. As a result of this enhanced photon environment, the radiative decay occurring in the perovskite is maximized and the PL improved. Further, reabsorption issues are reduced with this configuration as the largest fraction of light is generated closer to the exit side of the device. On the contrary, the worst example depicted in Fig.4f shows how, in this case, only a fraction of the emitters closer to the top part of the active layer contribute with an enhanced emission to the luminescent properties of the device.

While, as mentioned above, several factors should be considered when deciding the thickness of the different layers, from the possibility of growing homogeneous films to the carrier transport properties of the final device, here we have demonstrated how small variations can strongly influence the optical environment of the emissive layer through the density of states at its position. In particular, with a $\pm 20 \%$ thickness variation, an overall PL intensity enhancement of $11 \%$ integrated over all wavelengths and angles can be obtained with respect to the published device (18\% with respect to the worst device). Further, as the position of the emitter will also determine the way light exits the structure, the different proposed structures present strong variations in the angular pattern of the exiting light. This is reflected in the $16 \% \mathrm{PL}$ enhancement within a $5^{\circ}$ cone around normal incidence compared to the proposed structure (27\% if we compare it with the worst device) and if we compare the angular patterns of emitted light from all structures (see Fig.S4). This last point is a consequence of the fact that for the best scenario device the highest PL enhancement comes from a spatial region within the active layer for which light extraction close to normal incidence is optimized. While we have concentrated on an enhancement of the total radiated power, here it must be noted that one can refer to the optical environment of the active layer as a means to tailor directionality. Further, better improvements can be attained with larger thickness variations, depending on the flexibility of the fabrication procedure.

The strong influence that the local optical environment has on the dynamics of the photoemission process, as established by equation (1), is at the origin of the significant spectral 
variations observed on the luminescence extracted from the emitting layers of nanometric size as a function of their location. This implies that all emission coming from a light emitting device containing an arbitrarily thick active layer will be necessarily inhomogeneous, as it can be considered as the sum of the contribution of active regions exposed to many different optical environments, even if the emitting species is always and strictly the same all throughout the slab. This has relevant repercussions on the analysis of the emission spectrum and luminescence decay lifetime attained from any light emitting device and should always be considered.

Finally we would like to highlight that a similar optimization procedure can be carried out for other LEDs comprising thin active layers as is the case for QD-LEDs as summarized in Section S7 of the Supplementary Information.

A new generation of optoelectronic devices is being actively studied and reports from the past few years point to the possibility of reaching the performance of state of the art devices in the near future. These new systems will bring about the possibility of solution processing as well as new functionalities, but will likely demand the introduction of thin active layers in the range of tens of nanometers. Thus, in order to optimize their performance a careful design of their photonic environment will be necessary so that absorption/emission of light is maximized. As the final performance of the device will be affected by both light-matter interaction and charge transport, the present protocol could be further combined with finite element simulations in order to design devices which constituent layer thickness allow for optimized optical and electrical properties. Similar combined analysis have been employed in the past for QD, [41] organic [42] or conventional semiconductor solar cells [43] extending in some cases the analysis to heat flow effects. [44]

\section{ASSOCIATED CONTENT:}

Supporting information: FDTD simulation parameters; PL from dipoles located close to the edges of the emitting layer in the metallic cavity; Thickness of all simulated devices; Tailoring 
the local optical environment in perovskite solar cells; High efficiency perovskite-based LEDs; Angular distribution of PL from simulated LEDs; Tailoring the local optical environment in QD-LEDs.

\section{AUTHOR INFORMATION:}

Corresponding author: *Hernán Míguez, h.miguez@csic.es

Present address: ${ }^{\S}$ Max Planck Institute for Solid State Research, Heisenbergstraße 1, 70569 Stuttgart, Germany.

\section{ACKNOWLEDGEMENTS:}

The authors gratefully acknowledge Dr. M. Bernechea for providing spectrospic measurements from which optical constants of their fabricated device were retrieved. The research leading to these results has received funding from the European Research Council under the European Union's Seventh Framework Programme (FP7/2007-2013)/ERC grant agreement $n^{\circ} 307081$ (POLIGHT) and the Spanish Ministry of Economy and Competitiveness under grants MAT2014-54852-R and MAT2017-88584-R. A.J.-S. acknowledges the Spanish Ministry of Economy and Competitiveness for funding through an FPI program under the project MAT2011-23593.

\section{References:}

\footnotetext{
${ }^{1}$ Wiesmann, C.; Bergenek, K.; Linder, N.; Schwarz, U. T. Photonic Crystal LEDs - Designing Light Extraction. Laser \& Photon. Rev. 2009, 3, 262-286.
} 
${ }^{2}$ Atwater, H. A.; Polman, A. Plasmonics for Improved Photovoltaic Devices. Nat. Mater. 2010, 9, 205-213.

${ }^{3}$ Leung, S-F.; Zhang, Q.; Xiu, F.; Yu, D.; Ho, J. C.; Li, D.; Fan, Z. Light Management with Nanostructures for Optoelectronic Devices. J. Phys. Chem. Lett. 2014, 5, 1479-1495.

${ }^{4}$ Li, Y. Molecular Design of Photovoltaic Materials for Polymer Solar Cells: Toward Suitable Electronic Energy Levels and Broad Absorption. Acc. Chem. Res. 2012, 45, 723-733.

${ }^{5}$ Roncali, J.; Leriche, P.; Blanchard, P. Molecular Materials for Organic Photovoltaics: Small is Beautiful. Adv. Mater. 26, 3821-3838.

${ }^{6}$ Hu, L.; Kim, H. S.; Lee, J.-Y.; Peumans, P.; Cui, Y. Scalable Coating and Properties of Transparent, Flexible, Silver Nanowire Electrodes. ACS Nano 2010, 4, 2955-2963.

${ }^{7}$ Gaynor, W.; Hofmann, S.; Christoforo, M. G.; Sachse, C.; Mehra, S.; Salleo, A.; McGehee, M. D.; Gather, M. C.; Lüssem, B.; Müller-Meskamp, L.; Peumans, P.; Leo, K. Color in the Corners: ITO-Free White OLEDs with Angular Color Stability. Adv. Mater. 2013, 25, 40064013.

${ }^{8}$ Han, T.-H.; Lee, Y.; Choi, M.-R.; Woo, S.-H.; Bae, S.-H.; Hong, B. H.; Ahn, J.-H.; Lee, T.-W. Extremely Efficient Flexible Organic Light-Emitting Diodes with Modified Graphene Anode. Nat. Photonics 2012, 6, 105-110.

9 Sun, Z.; Chang, H. Graphene and Graphene-like Two-Dimensional Materials in Photodetection: Mechanisms and Methodology. ACS Nano 2014, 8, 4133-4156.

${ }^{10}$ Liu, Z.; Ping, S.; Yan, F. Functionalized Graphene and other Two-Dimensional Materials for Photovoltaic Devices: Device Design and Processing. Chem. Soc. Rev. 2015, 44, 5638-5679.

11 Balis, N.; Stratakis, E.; Kymakis, E. Graphene and Transition Metal Dichalcogenide Nanosheets as Charge Transport Layers for Solution Processed Solar Cells. Materials Today 2016, 19, 580-594.

${ }^{12}$ Novotny, L.; Hecht, B. Principles of Nano-Optics; Cambridge University Press: Cambridge, U.K., 2006. 
${ }^{13}$ Cho, H.; Jeong, S.-H.; Park, M.-H.; Kim, Y.-H.; Wolf, C.; Lee, C.-L.; Heo, J. H.; Sadhanala, A.; Myoung, N.; Yoo, S.; Im, S. H.; Friend, R. H.; Lee, T.-W. Overcoming the Electroluminescence Efficiency Limitations of Perovskite Light-Emitting Diodes. Science $\mathbf{2 0 1 5}, 350,1222-1225$.

${ }^{14}$ Xiao, Z; Kerner, R. A.; Zhao, L.; Tran, N. L.; Lee, K. M.; Hoh, T.-W.; Scholes, G. D.; Rand, B. P. Efficient Perovskite Light-Emitting Diodes Featuring Nanometer-Sized Crystallites. Nat. Photonics 2017, 11, 108-116.

${ }^{15}$ Yang, Y.; Zheng, Y.; Cao, W.; Titov, A.; Hyvonen, J.; Manders, J. R.; Xue, J.; Holloway, P. H.; Qian, L. High-Efficiency Light-Emitting Devices Based on Quantum Dots with Tailored Nanostructures. Nat. Photonics 2015, 9, 259-266.

${ }^{16}$ Sun, Q.; Wang, Y. A.; Li, L. S.; Wang, D.; Zhu, T.; Xu, J.; Yang, C.; Li, Y. Bright, Multicoloured Light-Emitting Diodes Based on Quantum Dots. Nat. Photonics 2007, 1, 717722.

${ }^{17}$ Lan, X.; Voznyy, O.; Pelayo García de Arquer, F.; Liu, M; Xu, J.; Proppe, A. H.; Walters, G.; Fan, F.; Tan, H.; Liu, M.; Yang, Z.; Hoogland, S.; Sargent, E. H. 10.6\% Certified Colloidal Quantum Dot Solar Cells via Solvent-Polarity-Engineered Halide Passivation. Nano Lett. 2016, 16, 4630-4634.

${ }^{18}$ Bernechea, M.; Miller, N. C.; Xercavins, G.; So, D.; Stavrinadis, A.; Konstantatos, G. Solution-Processed Solar Cells Based on Environmentally Friendly $\mathrm{AgBiS}_{2}$ Nanocrystals. Nat. Photonics 2016, 10, 521-526.

${ }^{19}$ Shin, S. S.; Yeom, E. J.; Yang;, W. S.; Hur, S.; Kim, M. G.; Im, J.; Seo, J.; Noh, J H.; Seok, S. I. Colloidally Prepared La-Doped $\mathrm{BaSnO}_{3}$ Electrodes for Efficient, Photostable Perovskite Solar Cells. Science 2017, 356, 167-171.

${ }^{20}$ Shen, L.; Fang, Y.; Wang, D.; Bai, Y.; Deng, Y.; Wang, M.; Lu, Y.; Huang, J. A SelfPowered, Sub-nanosecond-Response Solution-Processed Hybrid Perovskite Photodetector for Time-Resolved Photoluminescence-Lifetime Detection. Adv. Mater. 2016, 28, 10794-10800. 
${ }^{21}$ Tang, X.; Tang, X.; Lai, K. W. C. Scalable Fabrication of Infrared Detectors with Multispectral Photoresponse Based on Patterned Colloidal Quantum Dot Films. ACS Photonics. 2016, 3, 2396-2404

${ }^{22}$ Shi, Z.; Guo, J.; Chen, Y.; Li, Q.; Pan, Y.; Zhang, H.; Xia, Y.; Huang, W. Lead-Free Organic-Inorganic Hybrid Perovskites for Photovoltaic Applications: Recent Advances and Perspectives. Adv. Mater. 2017, 29, 1605005.

${ }^{23} \mathrm{http}: / /$ www.lumerical.com/tcad-products/fdtd/ (last accessed: 3 April, 2018)

${ }^{24}$ Rosencher, E.; Vinter, B. Optoelectronics; Cambridge University Press: Cambridge, U.K., 2004.

${ }^{25}$ For an updated report on perovskite solar cell certified efficiencies see the chart at: https://www.nrel.gov/pv/assets/images/efficiency-chart.png. (last accessed: 3 April, 2018)

${ }^{26}$ Anaya, M.; Lozano, G.; Calvo, M. E., Zhang, W.; Johnston, M. B.; Snaith, H.; Míguez, H. Optical description of Mesostructured Organic-Inorganic Halide Perovskite Solar Cells. $J$. Phys. Chem. Lett. 2015, 6, 85-99.

${ }^{27}$ Ball, J. M.; Stranks, S.D.; Hörantner, M. T.; Hüttner, S.; Zhang, W.; Crossland, E. J. W.; Ramirez, I.; Riede, M.; Johnston, M. B.; Friend, R. H.; Snaith, H. J. Low-Temperature Processed Meso-Superstructured to Thin-Film Perovskite Solar Cells. Energy Environ. Sci., 2015, 5, 602-609.

${ }^{28}$ Rahn, M.; Ma, D. Quantum-Dot-Based Solar Cells: Recent Advances, Strategies, and Challenges. J. Phys. Chem. Lett. 2015, 6, 85-99.

${ }^{29}$ Guo, K.; Lozano, G.; Verschuuren, M.; Gómez Rivas, J. Control of the External Photoluminescent Quantum Yield of Emitters Coupled to Nanoantenna Phased Arrays. $J$. Appl. Phys. 2015, 118, 073103.

${ }^{30}$ Johnson, P. B.; Christy, R. W. Optical Constants of the Noble Metals, Phys. Rev. B 1972, 6, 4370-4379. 
${ }^{31}$ Stelling, C.; Singh, C. R.; Karg, M.; König, T. A. F.; Thelakkat, M.; Retsch, M. Plasmonic Nanomeshes: their Ambivalent Role as Transparent Electrodes in Organic Solar Cells. Sci. Rep. 2017, 7, 42530.

${ }^{32}$ König, T. A. F.; Ledin, P. A.; Kerszulis, J.; Mahmoud, M. A.; El-Sayed, M. A.; Reynolds, J. R.; Tsukruk, V. V. Electrically Tunable Plasmonic Behavior of Nanocube-Polymer Nanomaterials Induced by a Redox-Active Electrochromic Polymer. ACS Nano 2014, 8, 61826192.

${ }^{33}$ Correa-Baena, J. P.; Anaya, M.; Lozano, G.; Tress, W.; Domanski, K.; Saliba, M.; Matsui, T.; Jacobsson, T. J.; Calvo, M. E.; Abate, A.; Grätzel, M.; Míguez, H.; Hagfeldt, A. Unbroken Perovskite: Interplay of Morphology, Electro-optical Properties, and Ionic Movement. $A d v$. Mater. 2016, 28, 5031-5037.

${ }^{34}$ Jeon, T.; Kim, S. J.; Yoon, J.; Byun, J.; Hong, H. R.; Lee, T.-W.; Kim, J.-S.; Shin, B.; Kim, S. O. Hybrid Perovskites: Effective Crystal Growth for Optoelectronic Applications. Adv. Energy Mater. 2017, 7, 1602596.

${ }^{35}$ Rakić, A. D. Algorithm for the Determination of Intrinsic Optical Constants of Metal Films: Application to Aluminum. Appl. Opt. 1995, 34, 4755-4767.

${ }^{36} \mathrm{Li}, \mathrm{H} . \mathrm{H}$. Refractive Index of Alkali Halides and its Wavelength and Temperature Derivatives. J. Phys. Chem. Ref. Data 1976, 5, 329-528.

${ }^{37}$ Wang, Z. B.; Helander, M. G.; Qiu, J.; Puzzo, D. P.; Greiner, M. T.; Hudson, Z. M.; Wang, S.; Liu, Z. W.; Lu, Z. H. Unlocking the Full Potential of Organic Light-Emitting Diodes on Flexible Plastic. Nat. Photonics 2011, 5, 753-757.

${ }^{38}$ Shibata, M.; Sakai, Y.; Yokoyama, D. Advantages and Disadvantages of Vacuum-Deposited and Spin-Coated Amorphous Organic Semiconductor Films for Organic Light-Emitting Diodes. J. Mater. Chem. C 2015, 3, 11178-11191.

${ }^{39}$ Born, M.; Wolf, E. Principles of Optics; Cambridge University Press: Cambridge, U.K., 1999.

${ }^{40}$ Potton, R. J. Reciprocity in Optics. Rep. Prog. Phys. 2004, 67, 717-754. 
${ }^{41}$ Miller, C. W.; Fu, Y.; Lopez, R. Enhancing Energy Absorption in Quantum Dot Solar Cells via Periodic Light-Trapping Microstructures. J. Opt. 2016, 18, 094002.

${ }^{42}$ Vervisch, W.; Biondo, S.; Rivière, G.; Duché, D.; Escoubas, L.; Torchio, P.; Simon, J.-J.; Le Rouzo, J. Appl. Phys. Lett. 2011, 98, 253306.

${ }^{43}$ Li, X.; Hylton, N. P.; Giannini, V.; Lee, K.-H.; Ekins-Daukes, N. J.; Maier, S. A. MultiDimensional Modeling of Solar Cells with Electromagnetic and Carrier Transport Calculations. Prog. Photovolt: Res. Appl. 2013, 21, 109-120.

${ }^{44}$ Shang, A.; Li, X. Photovoltaic Devices: Opto-Electro-Thermal Physics and Modeling. Adv. Mat. 2017, 29, 1603492. 